\title{
10 Jahre Praxisassistenzprogramm im Kanton Bern
}

\author{
Zsofia Rozsnyai ${ }^{a, c}$, Beatrice Dialloc, Sven Streit ${ }^{b, c}$

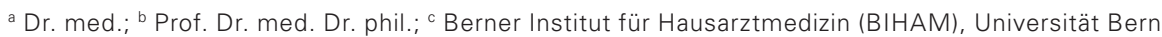

Disclosure statement Diese Studie wurde unterstützt von der Berner Stiftung zur Förderung der Hausarzt-Medizin (HAST, www.hast-bern.ch).

Dank

Dieser Artikel ist im doc.be 2/19 erschienen. Der Abdruck erfolgt mit freundlicher Genehmigung der Ärztegesellschaft des Kantons Bern.

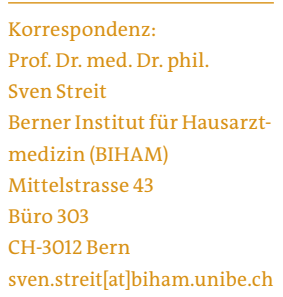

Was 2008 als Modellversuch im Kanton Bern begann, etablierte sich bis heute zum Standard in der Weiterbildung zur Hausärztin bzw. zum Hausarzt: die Praxisassistenz (PA). Junge Kolleginnen und Kollegen können während der PA hausärztliche Kenntnisse und Arbeitsweisen erlernen und verstehen, was der Hausarztberuf bedeutet. In den letzten zehn Jahren koordinierte das Berner Institut für Hausarztmedizin (BIHAM) mehr als 160 Praxisassistenzen. Seit 2019 ist das PA-Programm im Kanton Bern mit 35 Stellen das schweizweit grösste. Verantwortlich für diese Entwicklung waren Pioniere am BIHAM, die das Programm entwarfen, und in der Folge die kantonalen Verbände, welche das Projekt unterstützten.

\section{Gemeinsam gegen den Hausärztemangel}

Die Ärztegesellschaft des Kantons Bern (BEKAG) kämpfte Seite an Seite mit dem BIHAM, dem Verein Berner Hausund Kinderärzte (VBHK) und der Stiftung zur Förderung der Weiterbildung in Hausarztmedizin (WHM), um das Programm fortzuführen. Als es darum ging, das PA-Programm auszubauen, um dem Hausärztemangel entgegenzuwirken, unterstützte die BEKAG das BIHAM in den mitunter schwierigen Verhandlungen. An der BEKAGMittagsveranstaltung für Mitglieder des Grossen Rates und an weiteren Anlässen brachte die BEKAG die Forderungen aufs politische Parkett. Schliesslich stimmte der Grosse Rat 2017 einstimmig der Weiterführung und einem Ausbau des PA-Programms zu. Für die Periode 2019-2022 wurde die Anzahl Stellen von 21 auf 35 erhöht und zudem ein spezieller Fonds für unterstützungsbedürftige Praxen eingerichtet.

Die BEKAG unterstützt das BIHAM auch bei der Umsetzung des Programms. Vizepräsident Rainer Felber nahm Einsitz in das Board Praxisassistenz. Dieses hat gemeinsam mit dem Kantonsarztamt die Kriterien für die Vergabe des neu geschaffenen Unterstützungsfonds definiert und entscheidet darüber, wer eine PA-Stelle erhält.

\section{Befragung ehemaliger Praxisassistenten}

Wir nahmen das Jubiläum zum Anlass, alle 165 ehemaligen Praxisassistenten, welche von 2008 bis 2017 eine
PA begannen, zu befragen. Die wichtigste Frage war, ob sie bereits in eine Praxis eingestiegen sind. Es gelang, dies und weitere Informationen von über $90 \% \mathrm{zu}$ erfahren (siehe Abbildung).

Von den Teilnehmenden sind bereits mehr als $80 \%$ in der Praxis, haben den Einstieg geplant oder sind unterwegs dahin. Dies ist als grosser Erfolg zu werten. Wir waren aber erstaunt, dass über $40 \%$ dort eingestiegen sind, wo sie ihre PA absolviert hatten. Da wir am BIHAM bei der Zusage von PA-Gesuchen auch auf eine ausgewogene Verteilung im Kanton achten, können wir den Praxiseinstieg in Gebieten mit höherem Hausärztemangel gezielt unterstützen: In der Grafik sieht man, dass sich die Praxiseinstiege über den ganzen Kanton Bern verteilen. Verglichen mit der WorkforceStudie vom Institut für Hausarztmedizin in Basel (2015) zeigt sich, dass vor allem dort neue Praxen entstehen, wo der Mangel im Jahr 2015 am grössten war.

Eine weitere Frage war, was die jungen Ärztinnen und Ärzte in die Praxis bewegt. 87\% sagten die PA, 70\% die Lehrpraktikerinnen und Lehrpraktiker. Weitere Gründe waren die medizinische Vielfalt, eine langfristige Patientenbetreuung sowie die gute Vereinbarkeit von Beruf und Familie. Von denen, die nicht in die Praxis einstiegen, entschieden sich $10 \%$ für einen anderen Beruf oder unterbrachen ihre Arbeitstätigkeit zur Betreuung ihrer Kinder. Das BIHAM bietet bei Bedarf auch Beratung zur Unterstützung beim Wiedereinstieg an.

\section{Das PA-Programm zeigt Wirkung}

Das Berner PA-Programm bleibt eine Erfolgsgeschichte und gibt dem Kanton die Möglichkeit, seine Ressourcen so einzusetzen, dass es auch in ländlichen Gebieten zur Eröffnung neuer Hausarztpraxen kommt. Wir wissen heute, dass das Interesse an der Hausarztmedizin bei den Studierenden deutlich zugenommen hat. Diesen eine PA zu vermitteln wird der Weg bleiben, um dem Hausärztemangel entgegenzutreten, der sich mittelfristig aufgrund der Pensionierungen noch verstärken wird. Gemeinsam mit der BEKAG, dem VBHK und der WHM arbeiten wir daran, dass wir diese Durststrecke überstehen können und dass diese ein Ende nimmt. 


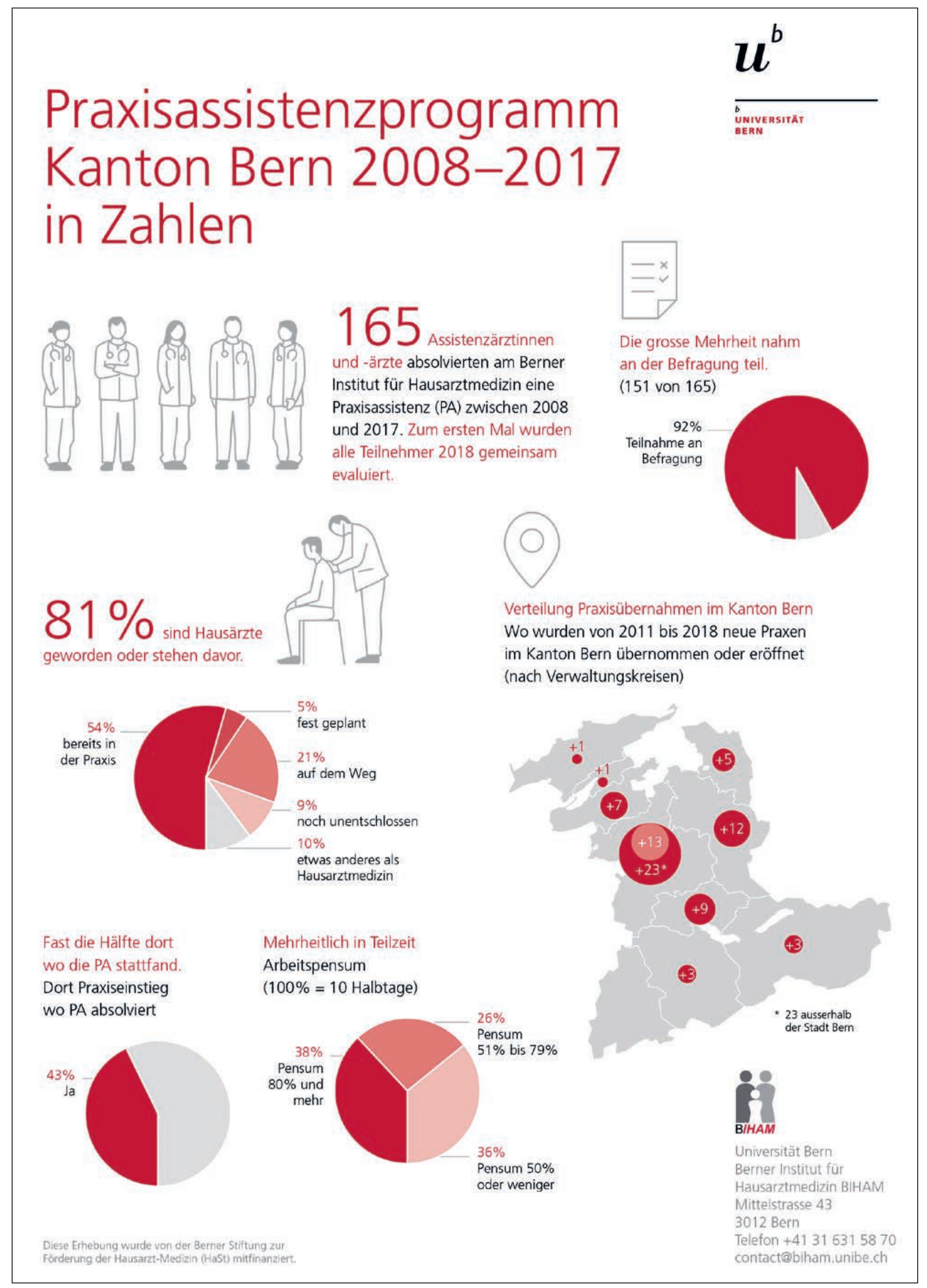

